\title{
ARTÍCULOS
}

\section{TENDENCIAS EN EL PERFIL TECNOLÓGICO DEL PROFESIONAL DE LA INFORMACIÓN}

\author{
Trends in the technological profile of information \\ professionals
}

\author{
Jorge Morato, Sonia Sánchez-Cuadrado y María-Teresa Fernández-Bajón
}

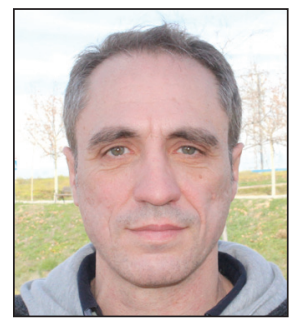

Jorge Morato, doctor en documentación por la Universidad Carlos III de Madrid, es profesor en el Departamento de Informática de dicha universidad, donde imparte docencia de grado y postgrado en informática y documentación. Es miembro del Instituto de Investigación Historiográfica Caro Baroja. Sus publicaciones y proyectos de investigación se centran en gestión del conocimiento, posicionamiento web, perfiles profesionales y recuperación de información web. http://orcid.org/0000-0002-7530-9753

Universidad Carlos III, Escuela Politécnica, Departamento de Informática Avda. Universidad, 30. 28911 Leganés (Madrid), España jmorato@inf.uc3m.es

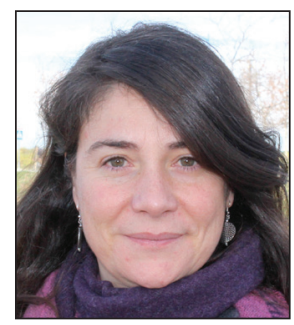

Sonia Sánchez-Cuadrado es doctora en documentación y trabaja en el Departamento de Biblioteconomía y Documentación de la Universidad Complutense de Madrid. Su actividad investigadora está enfocada a la extracción de información, reconocimiento de patrones, procesamiento del lenguaje natural, sistemas de organización del conocimiento y recuperación de información. También desempeña actividades de asesoría y gestión de elearning. http://orcid.org/0000-0002-7722-1982

Universidad Complutense, Facultad de Ciencias de la Documentación, Departamento de Biblioteconomía y Documentación Santísima Trinidad, 37. 28010 Madrid, España sscuadrado@ucm.es

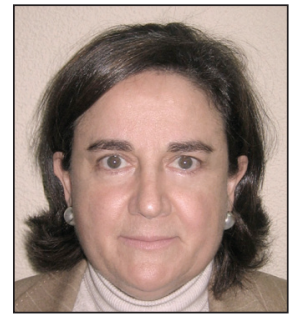

María-Teresa Fernández-Bajón, doctora en ciencias de la información por la Universidad Complutense de Madrid, es directora del Departamento de Biblioteconomía y Documentación de dicha universidad y directora del grupo de investigación Politecom. Sus principales líneas de investigación están centradas en las políticas de información, metodología y evaluación de la investigación. http://orcid.org/0000-0001-8067-4862

Universidad Complutense, Facultad de Ciencias de la Documentación Departamento de Biblioteconomía y Documentación Santísima Trinidad, 37. 28010 Madrid, España mtfernan@ucm.es

\section{Resumen}

Las tecnologías obligan a actualizar las competencias profesionales. Este estudio analiza estos cambios, tratando de aportar pautas para la mejora curricular. En este trabajo hemos realizado un análisis sobre los términos utilizados en 20 curricula de profesionales de la información y documentación para identificar sus competencias tecnológicas. Esas habilidades tecnológicas y esos términos han sido utilizados para examinar 735 ofertas de trabajo publicadas en portales generalistas y para 170 ofertas específicas. De este análisis hemos identificado los conocimientos y competencias que deben favorecer la inserción laboral de estos profesionales. Estos están relacionados principalmente con el marketing, la gestión y el software para publicación web. Los resultados confirman la tendencia positiva en la demanda laboral de las competencias tecnológicas atribuibles a los profesionales de la documentación. No obstante es necesario reclamar el reconocimiento de la profesión pues muchas de esas ofertas están clasificadas en otras especialidades. Asimismo aportamos una lista de conocimientos tecnológicos clave para los profesionales de la información y la documentación. La informática y la Web sin duda les proporcionan nuevas oportunidades. 


\title{
Palabras clave
}

Competencias profesionales; Perfiles profesionales; Mercado de trabajo; Profesionales de la información; Bibliotecarios; Documentalistas; Científicos de la información; Especialistas en información; Gestión de información; Tecnología.

\begin{abstract}
New technologies require an upgrade of LIS professionals' skills. This study analyzes changes in the field and suggests guidelines to improve LIS curriculum. We have carried out a content analysis of 20 curricula from LIS educational programs to identify terms associated with technological skills. In addition, we identified terms related to new competences from 735 job openings published on generic web sites and 170 on specific web sites. These terms include marketing, management, and content management, mainly related to web applications. The results confirm a positive trend in the demand for technological skills related to LIS. Nevertheless, there is a need to increase awareness about the competences and abilities of LIS professionals, because many relevant job openings are listed in other job categories. Therefore, we have collected a list of key technological skills. In conclusion, it is clear that computer science and the Internet are bringing new opportunities to LIS professionals.
\end{abstract}

\section{Keywords}

Professional skills; Professional profiles; Employment market; Information professionals; Librarians; Documentalists; Information managers; Information scientists; Information specialists; Information management; Technology.

Morato, Jorge; Sánchez-Cuadrado, Sonia; Fernández-Bajón, María-Teresa (2016). "Tendencias en el perfil tecnológico del profesional de la información". El profesional de la información, v. 25, n. 2, pp. 169-178.

http://dx.doi.org/10.3145/epi.2016.mar.03

\section{Introducción}

En los últimos años en España se ha discutido mucho sobre las salidas laborales de los estudios en información y documentación. Entre otros temas se ha cuestionado el motivo del descenso de matriculaciones, la adaptación de los estudios a las ofertas laborales, y la existencia de demanda laboral para esta titulación.

El objetivo de este trabajo ha sido comprobar esta situación, tanto en formación como en oferta laboral, e identificar los perfiles laborales y competencias que demanda actualmente el mercado. Para ello se ha tratado de comprobar la reducción efectiva en el número de alumnos universitarios con respecto a la inserción laboral, analizando los datos que proporciona el Ministerio de Empleo (SEPE). Seguidamente se ha estudiado la demanda de los estudios de información y documentación fuera de España para comprender si se trata de un fenómeno local o internacional. A continuación se ha realizado un estudio sobre los términos utilizados en los currículos para conocer la evolución de la carrera profesional de los profesionales de la documentación y determinar sus competencias tecnológicas. Y por último se han examinado ofertas de trabajo en España publicadas en portales web para identificar las competencias más demandadas.

\subsection{Reducción en la demanda de estudios}

De las estadísticas proporcionadas por el MECD se ha recopilado el número de estudiantes de información y do- cumentación en España respecto al total de universitarios entre los años 2010-2015 (tabla 1). Según esta fuente el número total de universitarios matriculados en ese período se ha reducido un $2,1 \%$, mientras que el número de matriculados en el grado de información y documentación ha disminuido en un $35,1 \%$. Con estos datos se puede afirmar que los estudios universitarios en información y documentación han visto reducida su matrícula de forma muy acusada en los últimos años.

\subsection{Reducción de la oferta laboral}

En la situación de crisis económica de los útimos años, la profesión no ha sido un caso excepcional y ha sufrido una disminución de la oferta laboral. Así, en el sector concreto de las bibliotecas públicas durante el período 2009-2014 se redujo el personal de las bibliotecas públicas españolas en un $0,92 \%(M E C D, 2014)$. En la estimación se ha eliminado el dato de Cataluña por no estar adecuadamente actualizado. Respecto a esta Comunidad Autónoma y de acuerdo con el Servei de Biblioteques del Departament de Cultura de la Generalitat de Catalunya (SLPC, 2013; SLPC, 2014) el personal se ha incrementado en el mismo período un $16,5 \%$. Sin embargo para el siguiente año, el último informe del observatorio de las ocupaciones del Ministerio de Empleo y Seguridad Social (SEPE, 2015), sobre las ocupaciones con tendencia positiva en contratación en 2014, indica un leve aumento de contratos dados de alta para técnicos y asistentes de bibliotecas $(4,5 \%)$ por parte de la administración pú-

Tabla 1. Número de estudiantes de información y documentación en España respecto al total de universitarios

\begin{tabular}{|l|c|c|c|c|c|}
\hline & $\mathbf{2 0 1 5}$ & $\mathbf{2 0 1 4}$ & $\mathbf{2 0 1 3}$ & $\mathbf{2 0 1 2}$ & $\mathbf{2 0 1 1}$ \\
\hline Total universitarios & 1.361 .340 & 1.416 .827 & 1.434 .729 & 1.456 .783 & 1.425 .018 \\
\hline $\begin{array}{l}\text { Universitarios grado información } \\
\text { y documentación }\end{array}$ & 2.335 & 2.724 & 3.019 & 3.391 & 3.573 \\
\hline
\end{tabular}


blica. Es sin duda un dato positivo, aunque la oferta pública está muy por debajo de la demanda de otras titulaciones.

Según algunos estudios, en el período 2008-2013, la reducción de puestos ofertados a través de la lista de distribución para profesionales de bibliotecas y documentación IweTel ha sido del 83,3\% (Tejada-Artigas; Chacón-Jarén; MoreiroGonzález, 2014).

Otros portales de empleo generalistas orientados al mundo laboral privado muestran un número discreto de ofertas de empleo donde se requiere el perfil de bibliotecario y archivero como tal. Este freno a la demanda de perfil tradicional ha sido señalado en varias publicaciones (Tejada-Artigas, 2013; Moreiro-González, 2014) y se observa que la mayoría de los empleadores proceden del sector privado (GarcíaMarco, 2013). En otros casos, los portales clasifican las ofertas por perfiles y no recogen anuncios para la especialidad de información y documentación (Laboris.net).

Del informe del MECD de 2015 se obtuvo el porcentaje de empleabilidad según la afiliación a la Seguridad Social a los cuatro años de finalizar, para el curso 2009/10 (MECD, 2015). Los datos indican que la licenciatura en documentación tiene un índice de empleabilidad superior a la media del conjunto de egresados universitarios (tabla 2).

En cuanto a las condiciones laborales, el salario de estos licenciados es superior a la media en 7,8 puntos; y en cuanto a la duración del contrato y al grupo de cotización de la Seguridad Social es similar al promedio. Por lo tanto no se puede afirmar que este colectivo sufra más desempleo que otros profesionales universitarios (MECD, 2015).

\subsection{Situación de la oferta laboral en otros países}

En países como Australia con una larga tradición bibliotecaria, los datos sobre la situación de los estudios y el empleo reflejan para las bibliotecas una oferta de trabajo reducida pero estable, y un número de alumnos universitarios constante (ALIA, 2015).

Un análisis de las ofertas de empleo publicadas en JobList de la ALA (American Library Association), muestra que se solicita principalmente el perfil del bibliotecario, aunque marca una clara diferencia con el perfil de documentalista.

http://joblist.ala.org

Se han consultado asimismo para Estados Unidos los informes anuales de Forbes sobre los masters que proporcionan mejores oportunidades de empleo a largo plazo. Desde hace varios años (2011-2015), los estudios de información y documentación (antes biblioteconomía y documentación -ByD) se sitúan en una posición discreta en relación al resto de demandas profesionales. Este ranking mide la rentabilidad en la inversión de los estudios, que se determina en

Tabla 2. Porcentaje de empleabilidad tras el cuarto año después de graduarse según la Seguridad Social (estadísticas MECD)

\begin{tabular}{|l|c|c|c|c|}
\hline & $\mathbf{2 0 1 4}$ & $\mathbf{2 0 1 3}$ & $\mathbf{2 0 1 2}$ & $\mathbf{2 0 1 1}$ \\
\hline Diplomado en ByD & 57,0 & 56,4 & 48,5 & 40,1 \\
\hline Licenciado en documentación & 71,6 & 68,8 & 68,8 & 65,1 \\
\hline Media de todas las titulaciones & 64,4 & 58,6 & 55,6 & 43,4 \\
\hline
\end{tabular}

función de los ingresos que perciben. Utilizan la aplicación Payscale que permite comparar un sueldo con el de otras personas en puestos similares, y elabora estadísticas para determinar la rapidez con la que se espera que aumente el empleo entre 2008 y el 2018. Sabemos que el estudio no puede ser extrapolable a muchos otros países por su idiosincrasia y las políticas de información que aplican. Sin embargo, se considera interesante por cuanto los ingresos de un trabajo suelen estar en relación con la demanda de ese puesto y su grado de especialización.

Para los estudios de biblioteconomía y documentación, Katie Bardaro, economista principal de Payscale, señala lo siguiente:

“Bibliotecología y documentación [...] tiene una distribución de pago bimodal. Para los que se especializan en este grado y entran en biblioteconomía con la perspectiva tradicional, la remuneración y el crecimiento del empleo son bastante limitados. Sin embargo, para aquellos que se especializan en este grado y por ejemplo trabajan en taxonomías en empresas con grandes volúmenes de datos, o empresas que utilizan algoritmos de búsqueda, la remuneración y el crecimiento del empleo son ambos muy respetables" (Smith, 2013).

\section{Aumentarán las ofertas laborales para los profesionales de la información y la documentación con competencias tec- nológicas}

Como se puede ver, destaca la doble naturaleza de la especialidad:

- desde el punto de vista tradicional considera que tanto la retribución como la oferta laboral serán limitadas;

- desde una especialización más tecnológica augura una evolución de la oferta laboral y sueldo bastante respetable para los que persigan un trabajo más de tipo documentalista en empresas.

Por otra parte la comparación de los informes de Forbes de 2011 (Smith, 2011) y 2015 (Dill, 2015) refleja un descenso de los estudios en informática o ciencias de la computación de la posición primera en 2011 a la novena en 2015. Este descenso parece favorecer a otras disciplinas centradas en las tecnologías de gestión de la información, actualmente en el 60 puesto. Esta área de especialización engloba aspectos de marketing, gestión, informática y documentación. También este hecho se puede observar entre los diez cursos más demandados en 2015 en la plataforma Coursera (2015). Entre ellos están algunos cuyos contenidos se enseñan en el grado, como marketing digital, diseño interactivo o marketing en redes sociales.

De manera análoga, Linkedln ha realizado un estudio a escala mundial en su red profesional para determinar las 25 actividades más demandadas. En la imagen 1 se puede observar el ranking de las actividades relacionadas con el perfil de los profesionales de la información y la documentación en los años 2013 y 2014. En la parte izquierda vemos como en 2013 la lista estaba encabezada por las actividades: análisis estadístico y minería de datos; software de integración; 
y sistemas de bases de datos. En el ranking de las actividades más relevantes de 2014 (parte derecha de la imagen 1) pierden relevancia las actividades que se encontraban en las tres primeras descendiendo a las posiciones 5a, $18^{a}$ y 25 a respectivamente. Por otra parte, irrumpen como novedades marketing web social, colocándose en 1a posición y la programación web (13a) con respecto al año anterior. El resto cambia su posición en el ranking cobrando mayor importancia el desarrollo para móviles (2a posición), los lenguajes de programación como Perl/Python/ Ruby (4a posición) o el marketing digital (7o posición).

En el listado se observan temas y conocimientos que se imparten en el grado de información y documentación (Moreiro et al., 2009a; 2009b) y que se utilizan en la actividad laboral de estos profesionales. Las coincidencias son especialmente remarcables en sistemas de almacenamiento y gestión, inteligencia de negocios, marketing digital, software de integración o arquitectura web.

Con el objeto de conocer cómo estos cambios tecnológicos afectan a los profesionales del sector se ha realizado un estudio sobre los términos utilizados en los curriculum vitae (CV). En ellos se puede observar el perfil profesional que los propios usuarios presentan sobre ellos mismos. Por otra parte, se han analizado las ofertas de trabajo de España publicadas en los portales web para identificar las competencias tecnológicas más demandadas.

Frente a una demanda tradicional estable hay nuevas oportunidades tecnológicas en el área

\section{Perfil de los documentalistas en LinkedIn}

En primer lugar se ha analizado un conjunto de $20 \mathrm{CV}$ de profesionales de biblioteconomía y documentación públicos en la red profesional Linkedln. La muestra del estudio es reducida, básicamente porque la red profesional limita en sus condiciones de uso la posibilidad de acceder a un número elevado de profesionales. Y aunque fue solicitado con fines científicos no obtuvimos permiso para realizar un estudio más amplio. El objetivo es descubrir qué perfiles y aptitudes tecnológicas tienen los profesionales cuya especialidad formativa es la biblioteconomía y documentación. A partir de los CV se extraen los términos que usan para definir su perfil y las actividades tecnológicas que han desempeñado. Estos términos serán las semillas que posteriormente se utilizarán para localizar ofertas de empleo.

Los resultados indican que en la vertiente de las tecnologías de la información, estos profesionales trabajan fundamentalmente en gestión y edición de contenidos y marketing online como community managers o expertos en SEO/SEM. Los términos encontrados asociados a cada uno de estos perfiles son:

- community manager: social media, redes sociales, Google, account manager, social media strategist, responsable de marketing, internet, dinamización de la comunidad online, marketing y publicidad, y desarrollo web;

- experto en SEO/SEM: Google, social media, account manager, account strategist, business development, consultor de marketing digital, redes sociales, CRM (customer relationship management).

Estos resultados están en línea con otros estudios que proponen estas profesiones como nuevos perfiles que puede asumir el profesional de la información y documentación. Esta tendencia hacia la integración de tecnología informática en la gestión de la información y documentación ha sido anteriormente observada en otros estudios en España (Tejada-Artigas, 2013; Moreiro et al., 2009b; Sánchez-Cuadrado et al., 2010), incluyendo los conocimientos de marketing y gestión. En concreto en un estudio donde se analizaron 1.020 ofertas en España, procedentes de un sitio especializado en este perfil, se comprobó la alta demanda de perfiles tecnológicos, sobre todo de aquellos centrados en internet y en el uso de software específico (Sánchez-Cuadrado et al., 2010). También Yang, Chen y Sun (2012) observaron la aparición de nuevos perfiles a partir de 60 ofertas en biblioteconomía y documentación. 


\section{Ofertas laborales según las competencias de un documentalista}

Para analizar las opciones laborales de los profesionales de la información y la documentación desde el punto de vista tecnológico se han realizado otros dos estudios: con el portal de empleo Laboris.net, y con un grupo de ofertas de portales especializados.

\subsection{Oferta de Laboris.net}

Nos preguntamos si existen ofertas de empleo que según las competencias solicitadas podrían ser realizadas por documentalistas. Se han recopilado las 735 ofertas publicadas en el portal Laboris.net en noviembre de 2015 y se han analizado de forma manual. Para ello se han tenido en cuenta las competencias específicas de la titulación propuestas en el Libro blanco del título de grado en información y documentación (Aneca, 2004), y se han buscado términos relacionados en las ofertas de trabajo. Esto ha servido para corroborar que la oferta es relevante para el perfil de los profesionales en información y documentación. Por último se han agrupado las ofertas por las áreas en las que han sido publicadas por el portal de ofertas y se ha recogido el número total de ofertas para cada área de Laboris.net.

Como se observa en la tabla 3, en la clasificación "Nuevas tecnologías e internet", todas las ofertas publicadas han sido consideradas relevantes para el perfil de profesional en información y documentación. Las principales actividades que se indicaban en las ofertas han sido community manager/specialist, digitalización documental, generación de documentación para marketing e información y bases de datos. También existen competencias orientadas a la especialización tecnológica del perfil de los profesionales de la información y la documentación.

De los datos obtenidos es destacable también la proporción que existe en las ofertas (gráfico 1) clasificadas como periodismo y comunicación (20\%) y el espacio creado para el sector

Tabla 3. Análisis de ofertas de empleo y su relación con competencias específicas del grado en ByD

\begin{tabular}{|c|c|c|c|c|}
\hline $\begin{array}{c}\text { Área } \\
\text { Laboris.net }\end{array}$ & $\begin{array}{l}\text { \# Relev. para } \\
\text { documentación }\end{array}$ & $\begin{array}{l}\text { Total de } \\
\text { ofertas por } \\
\text { categoría }\end{array}$ & Términos en las ofertas & $\begin{array}{l}\text { Competencias específicas relacionadas } \\
\text { según el Libro Blanco (Aneca, 2005) }\end{array}$ \\
\hline $\begin{array}{l}\text { Nuevas tecnolo- } \\
\text { gías e internet }\end{array}$ & 8 & 8 & $\begin{array}{l}\text { - community manager/specialist; } \\
\text { - digitalización documental; } \\
\text { - generación de documentación para mar- } \\
\text { keting e información; } \\
\text { - bases de datos }\end{array}$ & $\begin{array}{l}\text { - interacción usuarios y clientes de la } \\
\text { información; } \\
\text { - evaluación de recursos de información; } \\
\text { - gestión de colecciones; } \\
\text { - elaboración y difusión de la informa- } \\
\text { ción }\end{array}$ \\
\hline Marketing & 14 & 101 & $\begin{array}{l}\text { - técnico en marketing en redes sociales; } \\
\text { - aplicaciones para marketing y redes } \\
\text { sociales; } \\
\text { - analíticas web; } \\
\text { - diseño web; } \\
\text { - social media manager; } \\
\text { - posicionamiento (SEO/SEM); } \\
\text { - business development manager; } \\
\text { - generar documentación e informes; } \\
\text { - apps móviles para marketing; } \\
\text { - adwords; } \\
\text { - gestores de contenido; } \\
\text { - marketing online }\end{array}$ & $\begin{array}{l}\text { - análisis y representación de informa- } \\
\text { ción; } \\
\text { - técnicas de marketing; } \\
\text { - técnicas comerciales; } \\
\text { - gestión microeconómica; } \\
\text { - informática; } \\
\text { - técnicas de diagnóstico y evaluación }\end{array}$ \\
\hline $\begin{array}{l}\text { Informática y } \\
\text { comunicaciones }\end{array}$ & 4 & 228 & $\begin{array}{l}\text { - analista de calidad de datos; } \\
\text { - inteligencia de negocio; } \\
\text { - digital project manager; } \\
\text { - ETL y bases de datos; } \\
\text { - creación de documentos sobre estándares } \\
\text { y protocolos; } \\
\text { - big data }\end{array}$ & $\begin{array}{l}\text { - informática; } \\
\text { - organización y almacenamiento de la } \\
\text { información; } \\
\text { - gestión de colecciones y fondos }\end{array}$ \\
\hline Administrativo & 3 & 296 & $\begin{array}{l}\text { - bases de datos; } \\
\text { - búsqueda de información; } \\
\text { - asistente de contenidos }\end{array}$ & $\begin{array}{l}\text { - búsqueda de información; } \\
\text { - elaboración y difusión de la informa- } \\
\text { ción }\end{array}$ \\
\hline $\begin{array}{l}\text { Periodismo y } \\
\text { comunicación }\end{array}$ & 3 & 15 & $\begin{array}{l}\text { - social media; } \\
\text { - community manager; } \\
\text { - creación de contenidos; } \\
\text { - selección de aspectos de interés; } \\
\text { - mantener contenidos; } \\
\text { - SEO/SEM; } \\
\text { - bases de datos; } \\
\text { - asistencia a la generación de informes; } \\
\text { - campañas de marketing y social media }\end{array}$ & $\begin{array}{l}\text { - interacción usuarios y clientes de la } \\
\text { información; } \\
\text { - organización y almacenamiento de la } \\
\text { información; } \\
\text { - gestión de colecciones; } \\
\text { - elaboración y difusión de la informa- } \\
\text { ción }\end{array}$ \\
\hline $\begin{array}{l}\text { Educación y } \\
\text { formación }\end{array}$ & 2 & 87 & $\begin{array}{l}\text { - teleformación } \\
\text { - generación de propuestas; } \\
\text { - organización de materiales cursos online }\end{array}$ & $\begin{array}{l}\text { - elaboración y difusión de la informa- } \\
\text { ción, } \\
\text { - técnicas de producción y edición }\end{array}$ \\
\hline
\end{tabular}


del marketing (14\%). Estos resultados corroboran que las competencias de los estudios de información y documentación son en efecto requeridas por el sector privado, pero no como una categoría diferenciada. La explicación a esto puede ser o bien que el número de ofertas no es suficientemente significativo para que el portal lo presente como una única categoría, o bien que no son unos estudios suficientemente conocidos, aspecto ya reseñado por algunos expertos (Martínez-Comeche et al., 2015). Por otra parte, estos datos proporcionan a los profesionales de la información y documentación las categorías afines para buscar ofertas de empleo como: nuevas tecnologías e internet, periodismo y comunicación, y marketing.

El desconocimiento que parece existir entre los empleadores sobre la naturaleza de los estudios en biblioteconomía y documentación queda patente en las observaciones de Martínez-Comeche et al. (2015). Estos investigadores analizaron 1.705 ofertas, concluyendo que según los empleadores, el grado de información y documentación se relacionaba con ofertas como la gestión de documentos, pero no de la gestión de la información, ámbito en el que se demandaban otras titulaciones.

\subsection{Ofertas de otros portales especializados}

Para poder identificar la situación actual de la demanda del perfil de información y documentación desde el punto de vista de las destrezas tecnológicas asociadas al mismo, se han buscado ofertas de trabajo específicas en portales de búsqueda de empleo relacionados con la especialidad. En concreto estos portales de búsqueda han sido:

- COIE de la Universidad Carlos III de Madrid;

- LinkedIn.

En octubre de 2015 se recopilaron 170 ofertas de trabajo

Tabla 4. Términos identificados en las ofertas y porcentaje de ofertas que lo contienen

\begin{tabular}{|c|c|c|c|c|c|}
\hline Términos & $\begin{array}{c}\% \text { de } \\
\text { ofertas }\end{array}$ & Términos & $\begin{array}{c}\% \text { de } \\
\text { ofertas }\end{array}$ & Términos & $\begin{array}{c}\% \text { de } \\
\text { ofertas }\end{array}$ \\
\hline SEM & 28,24 & Marketing campaigns & 4,71 & Account strategist & 2,35 \\
\hline Social media & 26,47 & Software development & 4,12 & Analyze big sets of data & 2,35 \\
\hline Redes sociales & 21,18 & Mobile marketing & 4,12 & Conocimientos de html & 2,35 \\
\hline Google & 16,47 & Gestor de contenidos & 4,12 & Licenciado en marketing & 2,35 \\
\hline Internet & 12,35 & Bases de datos & 4,12 & Search engine & 2,35 \\
\hline Marketing digital & 11,76 & Content management systems & 4,12 & Dinamización de la comunidad online & 1,76 \\
\hline Net & 11,18 & Account management & 3,53 & Mobile apps & 1,76 \\
\hline Analista programador & 10,59 & Campañas de publicidad & 3,53 & Administración de portales web & 1,76 \\
\hline Wordpress & 10,59 & Contenidos y su viralización & 3,53 & Data scientist & 1,76 \\
\hline Linkedln & 9,41 & Gestionar el blog corporativo & 3,53 & Diseño gráfico & 1,76 \\
\hline Google analytics & 8,82 & Gestionar los perfiles de Facebook & 3,53 & Programación y control del contenido & 1,76 \\
\hline Community manager & 7,65 & Gestión comunidad online & 3,53 & Document management & 1,18 \\
\hline BBDD & 7,65 & Programming skills & 3,53 & Microsoft Office & 1,18 \\
\hline CRM & 7,65 & Redacción atractiva de sus contenidos & 3,53 & Responsable del centro de documentación & 1,18 \\
\hline Fluent in English & 7,06 & Business development & 2,94 & Responsable de marketing digital & 1,18 \\
\hline Google adwords & 6,47 & Base de datos & 2,94 & Responsable de marketing y comunicación & 1,18 \\
\hline Web technologies & 6,47 & Big data technologies & 2,94 & Account manager & 0,59 \\
\hline Big data & 5,88 & Data analysis & 2,94 & Archivo & 0,59 \\
\hline ROI & 5,88 & Machine learning & 2,94 & Biblioteca & 0,59 \\
\hline Decision making & 5,88 & Gestión de contenidos & 2,94 & Gestor de proyectos & 0,59 \\
\hline Analytical skills & 5,88 & Posicionamiento web & 2,94 & Responsable de información & 0,59 \\
\hline Responsable de marketing & 4,71 & Desarrollo web & 2,35 & & \\
\hline
\end{tabular}




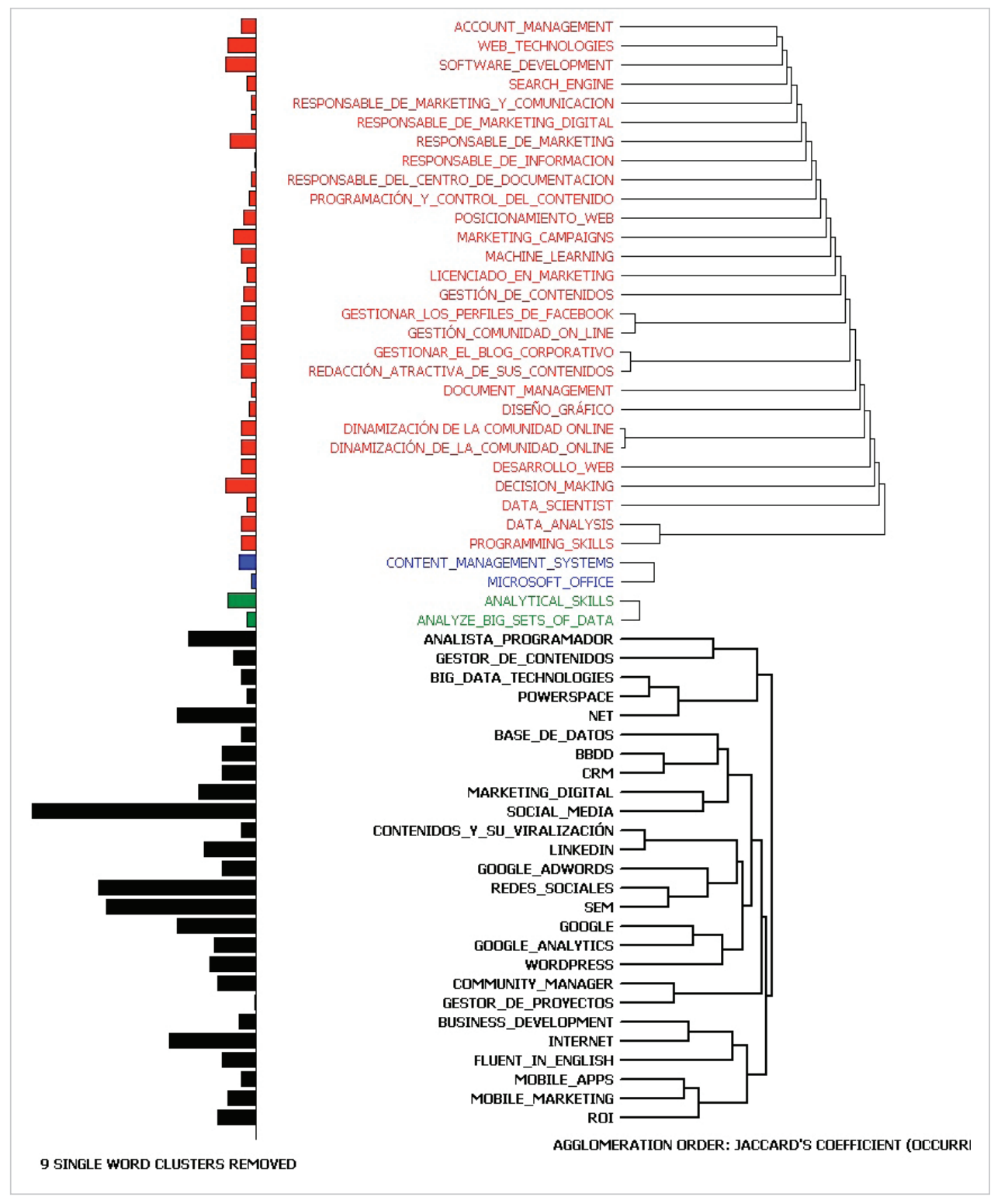

Grafico 2. Representación de los clusters resultantes de aplicar el coeficiente de Jaccard para los términos de las ofertas
4 recoge los resultados de análisis de las ofertas y los términos relacionados a contenidos afines a la especialidad y muestra el porcentaje de ofertas en las que han aparecido. Se observa una representación significativa de conceptos de carácter tecnológico.

Para identificar detalladamente la asociación de los conceptos localizados o, lo que es lo mismo, la relación de coocurrencia y proximidad de los términos en las ofertas de trabajo se muestra el gráfico 2 resultante de aplicar el coeficiente de Jaccard. Se puede observar cómo se han formado 4 grupos o clusters.

Otra forma de visualización es representando los datos como una red conceptual. El gráfico 3 refleja esa relación para el cluster 4. A nuestro juicio, en este gráfico 3 se aprecia la relevancia de las campañas de marketing online en relación con las herramientas asociadas para su promoción. Por otra parte también se refleja una relación significativa entre marketing digital y redes sociales. relacionadas con conocimientos tecnológicos de la especialidad. Las consultas para recuperar estas ofertas se realizaron a partir de los perfiles tecnológicos reseñados en la bibliografía y resultantes de nuestros estudios. Tras el análisis inicial de las ofertas se consideró oportuno incluir la denominación de algunas otras consultas. Los términos de estas búsquedas han sido:

- software: Alfresco, Nuxeo, Joomla, DSpace, Documentum, CMS;

- web semántica: linked data, RDF, OWL, metadatos;

- marketing: SEO/SEM, posicionamiento web, análisis de opinión, opinion analysis, branding, imagen de marca;

- perfiles: content curator/curador de contenidos, científico de datos/data scientist, social, gestor de contenidos, community manager;

- actividades documentales de tratamiento de datos: data cleansing, limpieza de datos, entidades del nombre.

En este caso, el método de análisis para las ofertas de empleo ha sido el análisis cualitativo de datos. Se ha calculado la frecuencia de los términos en los documentos. La tabla

\section{Conocimientos tecnológicos clave}

La realidad de la Web y las nuevas tecnologías tienen consecuencias evidentes en el mercado de trabajo provocando una ampliación hacia un contexto más actualizado del perfil profesional de la información y documentación. A la vista de los resultados de este estudio, la evolución de estos profesionales debe seguir incidiendo en el perfil tecnológico de manera que le proporcione mayor capacidad de adaptación. Desde este punto de vista no hablamos de una nueva disciplina sino de una actualización tecnológica de las herramientas. Al ser internet el gran repositorio de información parece evidente que buena parte de las técnicas deban centrarse en este contexto. Los conocimientos tecnológicos clave para el profesional están relacionados con:

- soportes tecnológicos para la gestión documental;

- gestión de contenido web en redes sociales;

- web semántica para productos de valor añadido;

- posibilidades de negocio con el tratamiento de la información. 


\section{Soporte tecnológico}

De forma similar a la evolución de los opacs que se popularizaron en los años 80 y se trasladaron a la Web en los 90, actualmente los gestores como Koha o Kobli-Koha para bibliotecas, ArchivesSpace o ICA-Atom para archivos, o $\mathrm{Nu}$ xeo, Alfresco o E-prints o DSpace para gestión de contenidos, hacen imprescindible un conocimiento básico de este tipo de software. La base más popular para este grupo de aplicaciones es LAMP (Apache, MySQL, $\mathrm{PHP})$. La instalación, configuración y personalización de estos gestores es un perfil con una demanda creciente, debido a que comparten un conjunto de tecnologías.

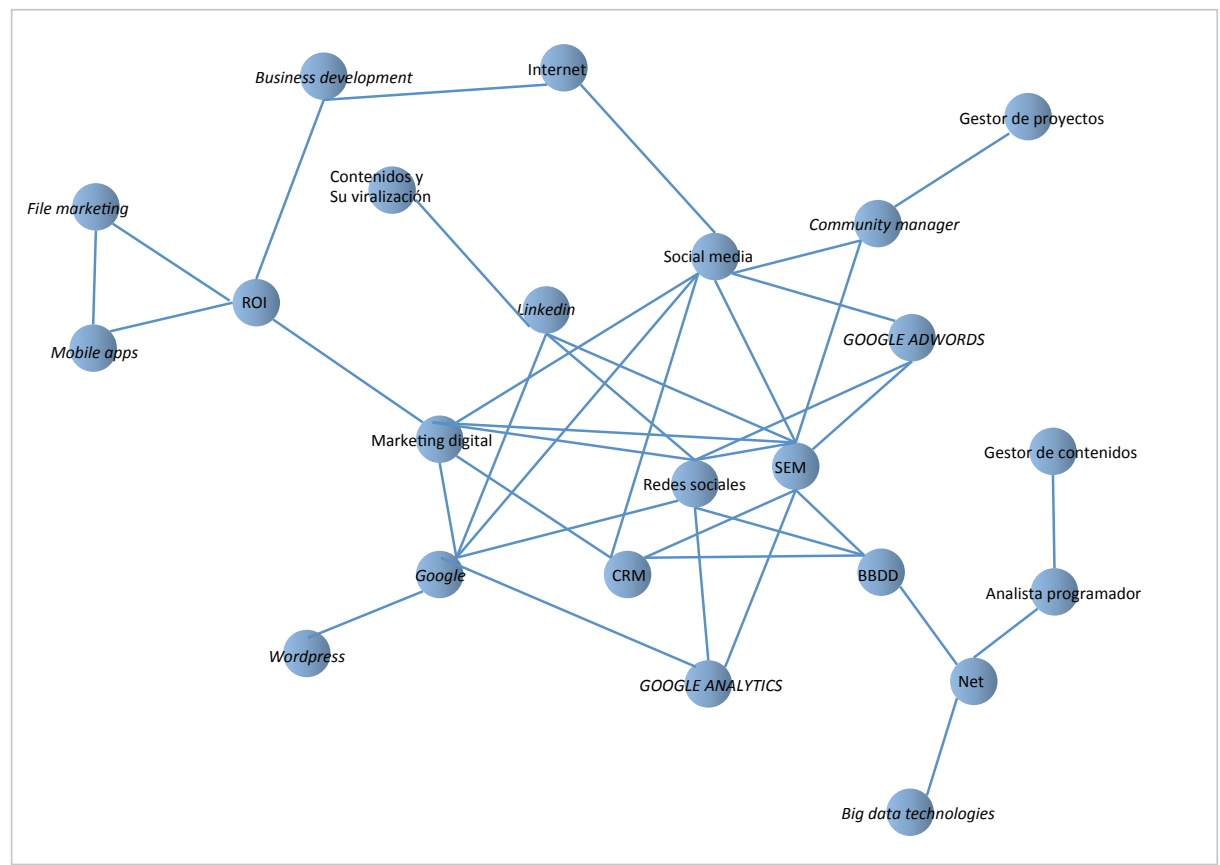

Grafico 3. Red conceptual del cluster 4 de las ofertas de trabajo

\section{Multiplicidad de plataformas}

La necesidad de que la información ofrecida al usuario pueda ser consultada en todo momento desde cualquier plataforma plantea el reto de la capacidad de poner a disposición del público información de forma clara y útil.

\section{Volumen de datos y de contenidos}

El gran volumen de información hace que la inclusión de sistemas de organización de información y anotación sea una necesidad. Esto se puede apreciar desde el uso de folksonomías hasta las ontologías. A este hecho se añade la gran potencia de la tecnología big data, que permite aprovechar el incremento de datos en abierto. De especial relevancia son los datos de investigación utilizados en determinados estudios. Distintos perfiles han surgido a raíz del volumen de contenidos. Por ejemplo el denominado content curator (Guallar, 2014), y uno más industrial y estadístico, el científico de datos, en el contexto de big data principalmente. Este último, aunque más técnico, comparte con el primero la necesidad de identificar fuentes de información/datos, evaluar su fiabilidad, mejorar la calidad y ver medios para su agregación, fusión o integración con otros recursos. Estos perfiles requieren de soporte tecnológico, principalmente de bases de datos NoSQL. Pero identificar relaciones entre conjuntos de datos, de forma similar a linked data es una de las diez grandes tendencias para los próximos años, tarea que debe ayudarse de técnicas de reconocimiento de entidades, procesamiento de lenguaje natural (PLN) e inteligencia artificial (Cearley; Walker; Burke, 2015).

\section{Fiabilidad de las fuentes}

La procedencia diversa de los datos y las informaciones, con distintos grados de fiabilidad y calidad, provoca la necesidad de que el análisis y selección de fuentes sea a cargo de especialistas.

\section{Gestión de contenidos en redes sociales}

El perfil de community manager es uno de los más demandados en la Web para evaluar contenidos y difundir información. Este tipo de perfil puede requerir rastrear información personal para su gestión en el futuro.

\section{Web semántica}

En el informe de Linkedln se señalaba la importancia del software de integración. La principal apuesta del mundo de las ciencias documentales es la generación de vocabularios de metadatos, sistemas de organización del conocimiento e interconexión mediante datos enlazados. Existen múltiples serializaciones de estos datos en la Web, si bien las tripletas están ganando importancia como unidad de información. Un adecuado tratamiento de estos datos parece ser una necesidad también en el presente de la profesión.

\section{Marketing digital y SEO son estrategias bá- sicas para poner en valor la información}

\section{Marketing}

La puesta en valor de la información, como un producto más para generar negocio, es clave en el desarrollo web de las técnicas de marketing en redes sociales, redireccionamiento web y posicionamiento en buscadores.

\section{Conclusiones}

No es cuestionable el hecho del descenso en el alumnado de la disciplina, sin embargo esto no puede justificar una falta de perspectivas laborales para los profesionales de la información y la documentación. Como ocurre en otros países, en España se mantiene una demanda laboral relativamente constante del perfil tradicional, mientas que una especialización tecnológica evoluciona de forma creciente. 
Los datos de los informes de distintas fuentes (Linkedln, Informe Gartner y Forbes) auguran que la demanda y necesidad futura de los profesionales de la información y documentación con competencias tecnológicas para el entono digital experimentará un fuerte repunte.

Las habilidades demandadas por los empleadores raramente coinciden con un único grado universitario, sino con una mezcla de habilidades de varias especialidades. Es por esto que las demandas deben ser analizadas por las competencias y conocimientos requeridos y su coincidencia con las habilidades adquiridas en los estudios, en vez de por la titulación demandada.

Tan sólo una pequeña muestra de CV deja ver cómo los profesionales se forman en competencias tecnológicas y llevan a cabo labores relacionadas con la gestión de contenido web, la gestión de datos y el marketing online.

Es necesario adaptarse a los nuevos contextos con el fin de facilitar al usuario una información accesible, relevante y de calidad. Es preciso no sólo actualizar el CV para completar el actual con las nuevas demandas, sino también difundir el potencial de la titulación entre los empleadores que precisan gestionar la información.

No hablamos de una nueva disciplina sino de una actualización tecnológica de las herramientas

\section{Nota}

Queremos agradecer a los revisores de la revista y a Natalia Arroyo-Vázquez sus aportaciones y sugerencias en la redacción del artículo.

\section{Bibliografía}

ALIA (2015). ALIA LIS education skills and employment. Trend report 2015. Australian Library and Information Association. https://www.alia.org.au/employment-and-careers/alia-liseducation-skills-and-employment-trend-report

Aneca (2004). Libro blanco del título de grado en información y documentación. Madrid. Agencia Nacional de Evaluación de la Calidad y Acreditación, p. 238.

http://www.aneca.es/modal_eval/docs/conver_biblio.pdf

Cearley, David-W; Walker, Mike J.; Burke, Brian (2015). Top 10 strategic technology trends for 2016: At a glance. Informe Gartner.

https://www.gartner.com/doc/3143618/top--strategictechnology-trends

Coursera (2015). "The most coveted Coursera Certificates". Coursera Blog.

http://coursera.tumblr.com/post/135338805637/our-top10-most-coveted-certificates-of-2015

Dill, Kathryn (2015). “The best and worst master's degree for jobs in 2015". Forbes, 15 July.

http://www.forbes.com/sites/kathryndill/2015/07/15/the-bestand-worst-masters-degrees-for-jobs-in-2015/\#146939e5561f
García-Marco, Francisco-Javier (2013). “Educación aprendizaje de la información y la documentación: raíces, desafíos y líneas de acción". El profesional de la información, v. 22, n.6, pp. 489-504.

http://dx.doi.org/10.3145/epi.2013.nov.01

Guallar, Javier (2014). “Curación de contenidos en la era de la infoxicación. Propuestas para bibliotecas". En: VII Jornadas de la Red de Bibliotecas del Instituto Cervantes, 11 diciembre. http://eprints.rclis.org/28645

Martínez-Comeche, Juan-Antonio; Carpallo-Bautista, Antonio; Burgos-Bordonau, Esther; Francisco-Olmos, JoséMaría (2015). "La demanda de profesionales de la información y documentación en España". Revista general de información y documentación, v. 25, n. 1, pp. 175-190. http://revistas.ucm.es/index.php/RGID/article/view/48984

MECD (2010-2015). Estadística de estudiantes. Madrid: Ministerio de Educación, Cultura y Deporte http://goo.gl/57chCN

MECD [2014]. Bibliotecas públicas españolas en cifras. Madrid: Ministerio de Educación, Cultura y Deporte

http://mecd.gob.es/cultura-mecd/areas-cultura/ bibliotecas/mc/ebp/portada.html

http://www.cultura.gob.es/alziraweb/alziraweb. $\mathrm{cmd}$ ?command $=$ GetAnexo\&id $=49$

MECD (2015). Inserción laboral de los egresados universitarios. La perspectiva de la afiliación a la Seguridad Social. Primer informe. Madrid: Ministerio de Educación, Cultura y Deporte; Conferencia de Consejos Sociales.

http://goo.gl/QgOMuB

Moreiro-González, José-Antonio (2014). “Aún en torno a la formación universitaria, el empleo y la recesión". Anuario ThinkEPI, v. 8, pp.15-23.

Moreiro-González, José-Antonio; Sánchez-Cuadrado, Sonia; Morato, Jorge; Tejada-Artigas, Carlos-Miguel (2009a). "Creación de un corpus coordinado de competencias en información y documentación a partir de ofertas de empleo en España". RISTI. Revista ibérica de sistemas y tecnologías de información, n. 3, pp. 79-91.

http://www.aisti.eu/risti/RISTI\%20N3.pdf

Moreiro-González, José-Antonio; Sánchez-Cuadrado, Sonia; Morato, Jorge; Moreno, Valentín (2009b). “Desarrollo de una aplicación ontológica para evaluar el mercado de trabajo español en biblioteconomía y documentación". Revista española de documentación científica, v. 32, n. 1, pp. 81-98. http://dx.doi.org/10.3989/redc.2009.1.675

Sánchez-Cuadrado, Sonia; Morato, Jorge; Andreadakis, Yorgos; Moreiro-González, José-Antonio. (2010). "A study of labour market information needs through employers seeking behaviour". Information research, v. 15, n. 4, paper 441. http://InformationR.net/ir/15-4/paper441.htm/

SEPE (2015). Año 2014: Ocupaciones con tendencia positiva en contratación. Ministerio de Empleo y Seguridad Social. Observatorio de las Ocupaciones. Servicio Público de Empleo Estatal.

https://goo.gl/tırZgr 
SLPC (2013). Sistema de Lectura Pública de Catalunya Balanç de 2013. Barcelona: Servei de Biblioteques del Departament de Cultura.

http://goo.gl/ZOzRKR

SLPC (2014). Biblioteques públiques de Catalunya Balanç de 2014. Barcelona: Servei de Biblioteques del Departament de Cultura.

http://goo.gl/FLLMr2

Smith, Jacquelyn (2013). "The best and worst master's degrees for jobs right now". Forbes, 7 June.

http://goo.gl/sQTGz9

Tejada-Artigas, Carlos-Miguel (2013). "Informe de situación: profesión y formación universitaria en 2012”. Anuario
ThinkEPI, v. 7, pp. 17-23

Tejada-Artigas, Carlos-Miguel; Chacón-Jarén, Sandra; Moreiro-González, José-Antonio (2014). “Mercado de trabajo en información y documentación y crisis económica en España: una aproximación a partir de las ofertas publicadas en IweTel entre 2008 y 2013". BiD: textos uni-versitaris de biblioteconomia i documentació, n. 32.

http://dx.doi.org/10.1344/BiD2014.32.15

Yang, Yuli; Chen, Tao; Sun, Jun (2012). "The impact of IT on job qualifications for librarians in the digital age and implications for LIS education". En: 2012 Int. symp. on information technology in medicine and education.

http://dx.doi.org/10.1109/ITIME.2012.6291260

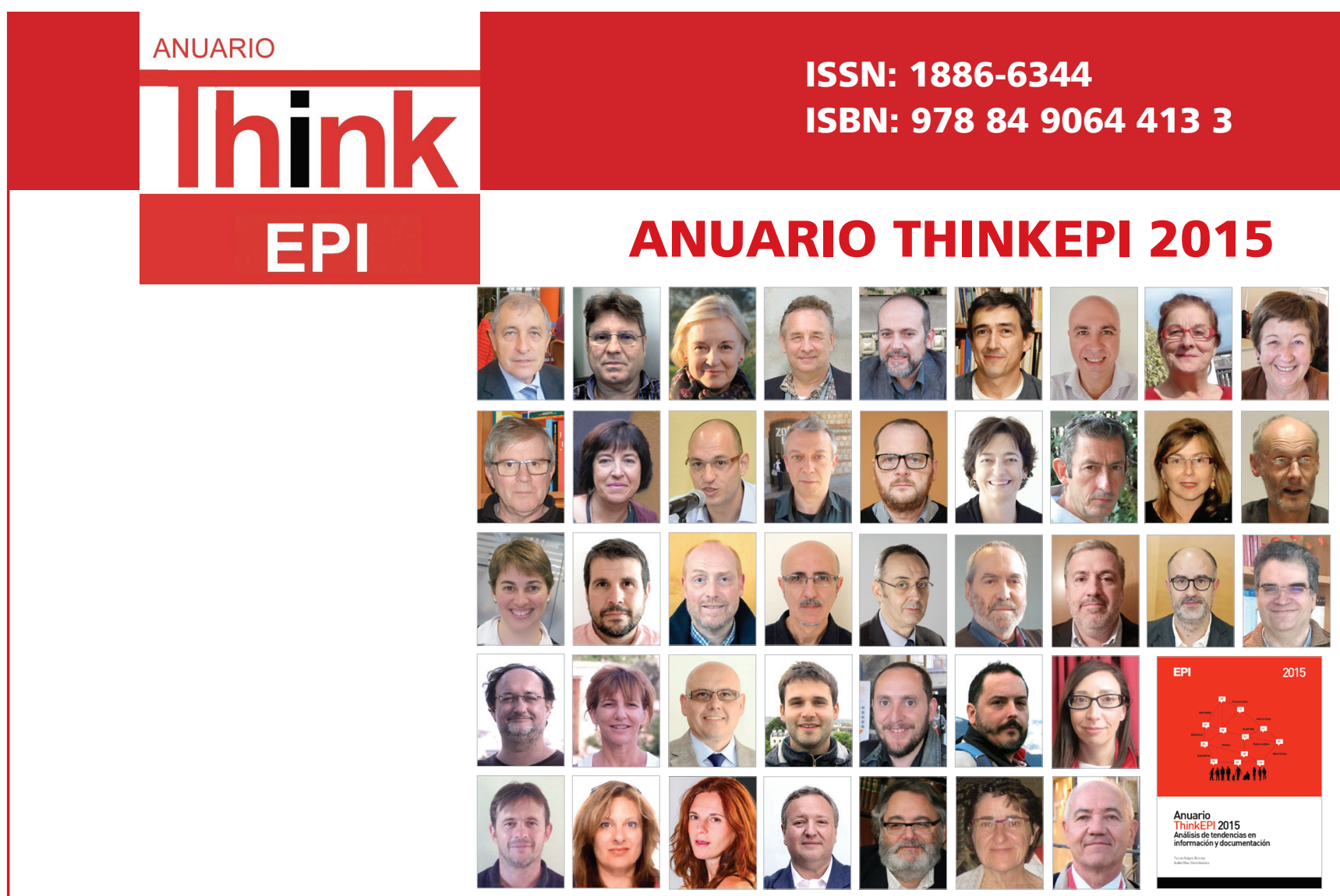

\section{PRECIOS ANUARIO THINKEPI}

Suscripción online (2007-2015)

Instituciones $75 €$

Individuos (particulares) $45 €$

Números sueltos

Instituciones

Anuario ThinkEPI 2015 (pdf) $55 €$

Anuario de años anteriores* ............ $30 €$ Individuos (particulares)

Anuario ThinkEPI 2015 (pdf) $30 €$

Anuario de años anteriores* $22 €$
Desde 2014 es posible el acceso mediante suscripción a todos los Anuarios ThinkEPI publicados hasta el momento desde el Recyt de la Fecyt

http://recyt.fecyt.es/index.php/ThinkEPI

\author{
Más información: \\ Isabel Olea \\ epi.iolea@gmail.com
}

